\title{
Holonic Modeling of Environments for Situated Multi-Agent Systems
}

\author{
Sebastian Rodriguez - Vincent Hilaire - Abder Koukam \\ Université de Technologie de Belfort-Montbèliard \\ Systems and Transports Laboratory \\ 90010 Belfort Cedex \\ FRANCE \\ sebastian.rodriguez@utbm.fr \\ tel: +33 384583837 - fax +33 384583342
}

\begin{abstract}
In a Multi-Agent Based Simulation (MABS) special attention must go to the analysis, modeling and implementation of the environment. Environments for simulation of real world problems may be complex. Seeing the environment as a monolithic structure only reduces our capacity to handle large scale, real-wold environments. In order to support this type of environments, we propose the use of an holonic perspective to represent the environment and the agents. In our approach, agents and environment are represented by holons. The environment defines an holarchy. Agents belong to a specific holon in this holarchy following their needs.
\end{abstract}

Key words: holonic modeling, environment of MAS, simulation

\section{Introduction}

It is generally accepted that multi-agent systems (MAS) operate within an environment $[11,9]$. In an Agent Based Simulation (ABS), special attention must go to the analysis, modeling and implementation of the environment [10]. Indeed, it simulates a real-world environment and agents represent acting entities in this environment.

Environments for simulation of real world problems may be complex. Indeed, a real world problem, as the one we present in this paper, is frequently characterized by an environment composed of heterogeneous and numerous entities. However, current practice of ABS modeling and simulation tends to consider the environment as monolithic structure. This approach, even if useful in certain situations, limits our capability to develop large scale agent based simulations.

Defined by Koestler [8] as entities that can not be considered as wholes nor parts in an absolute sense, Holons provide a possible answer to this problem. According to Koestler, a holon is a self-similar structure that consists of several holons as sub-structures. The hierarchical structure composed of holons is called 
holarchy. Holonic systems have already been used to model a wide range of systems, Manufacturing systems [2] Transportation [3], Adaptive Mesh Problem[13] and Cooperative work[1], to mention a few.

In order to show how holonic concepts can be applied to model and simulate large environments, we present in this paper a holonic based model for the traffic network of an important industrial plant of the east of France. The Peugeot SA (PSA) plant is located near two towns and directly connected to the highway and the railway. Within a surface of over 250 hectares, the plant produces more than 1700 cars per day. The plant can be seen as a small town with a high density of traffic that needs to be regulated. A simulator was built to detect possible bottle-necks and evaluate the plant's design. In order to produce a scalable and reliable traffic simulator, we must carefully model this environment to be both efficient and realist.

The use of holons to model both, environment and agents, is the natural consequence of seeing the environment as an active entity, and not merely as a passive component modified by agents at will. The environment is seen as an active entity, capable of interacting with agents and able to enforce the environmental principles [11].

This work aims to provide a new approach to model large scale environments. The interest of a holonic view of the environment is that it provides a scalable multi-level model to express real-world environments. This is achieved by using a hierarchical approach. The designer is able to represent different levels of detail, from a high-level coarse-grained view of the system to a low-level fine-grained one.

The paper is organized as follows : section 2 introduces the holonic environment model, section 3 discusses the simulation principles; eventually, section 4 presents related works and concludes.

\section{A Holonic model for Traffic Networks}

We propose the use of holarchies to model environments. In the PSA example, we want to simulate the traffic within the plant. The environment of this example is the traffic network, and its structure is defined by the topology of the plant. The agents will be the different vehicles driving through the plant.

The environment, represented by a holarchy, defines the organizational and topological structure. Each environmental holon represents a specific granularity level of the real plant's topology and enforces the environmental laws. This holarchy is static and predefined as it represents the real plant's environment. Indeed, the latter can't change at run-time, and the environmental laws (physic laws) are known a priori.

In order to represent the geographical environment of the plant as a holarchy, we have to find recursive concepts which represent the plant's components. The concepts we have chosen are described in the figures 1 and 2 .

Figure 1 shows that a road is divided into links. A link represents a one way lane of a road. A segment is composed of two exchange points, called input and 


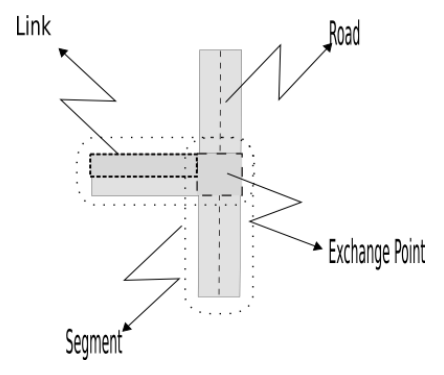

Fig. 1. Roads, Segments, Links and Exchange Points

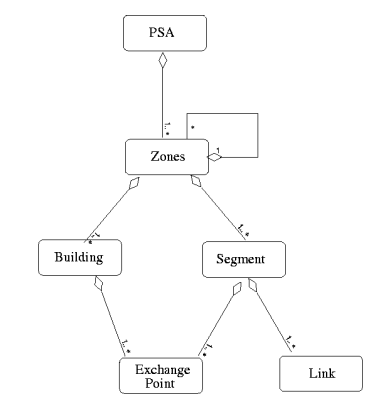

Fig. 2. Conceptual view of the plant

output exchange points, and, at least, one link. Exchange points let vehicles pass from one link to the other. And they are always shared by at least two segments.

Figure 2 presents the hierarchical decomposition of the environment of the plant. We can see that the industrial plant is composed of a set of zones, that contain Buildings and Segments. Buildings and Segments can also communicate through shared exchange points. Usually, an exchange point represents a crossroad, but it can also represent an entrance used by trucks to access buildings. A zone may also be recursively decomposed into smaller zones.

ExchangePoints are always shared by two segments or one segment and a building. As we can see the exchange point is a "special" role from the "holonic point of view" since the role is actually shared be more than one super-holon (Segment or Building) by definition.

Such a hierarchical decomposition of environments is based on the idea of Simon, who defines a nearly decomposable system (NDS) [15] as presenting two distinctive characteristics. The first characteristic is that the short-run behavior of each sub-component is approximately independent of the short-run behavior of other components. The second characteristic is that in the long run the behavior of any one of the components depends only in an aggregate way on the behavior of the other components. Based on this definition, we can define a Nearly Decomposable Environment (NDE) as the environment where we can find a decomposition that respects the propositions stated for NDS. Traffic Networks can be seen as a NDE, since in the short-run the behavior and phenomena that may exhibit a zone of the traffic remains independent of the behavior of other components. Indeed, phenomena like congestion, jams and others, remain localized in a zone before spreading.

This model presents several advantages when compared to a global representation. First, no size limit is imposed by the model. This enables us to use the same environment decomposition to simulate the traffic inside a city or a (much) smaller industrial plant. If required, semantic information can be introduced; so instead of zones, we will represent quarters, blocks, etc. [5].

Another interesting characteristic is that all necessary information to simulate the traffic inside a link is local (other vehicles, roadsigns, etc). This makes 
the model easier to distribute in a network and leaves the door open to Real-Time applications as well as Virtual Reality implementations.

In this work we have concentrated in the traffic network, but the decomposition of the environment may continue to provide a higher level of detail. For instance, a building can be decomposed in Rooms and Exchange Points(doors). The model provides a simple and flexible way to decompose different types of environments. Even more, it offers means for these different environments to coexist in the same simulation.

On the other hand, this type of decomposition imposes a highly hierarchical and decentralized representation of the environment. This could present some disadvantages when the environment presents some global "variables" accessible to all agents.

\section{Simulation}

As presented in section 2, the environment is modeled as a holarchy. Each environmental holon represents a specific context. In the PSA example, it is a specific place in the plant. These places have different granularity levels according to their level in the holarchy. During the simulation, vehicle agents move from one holon to another and the granularity is chosen using execution or simulation constraints.

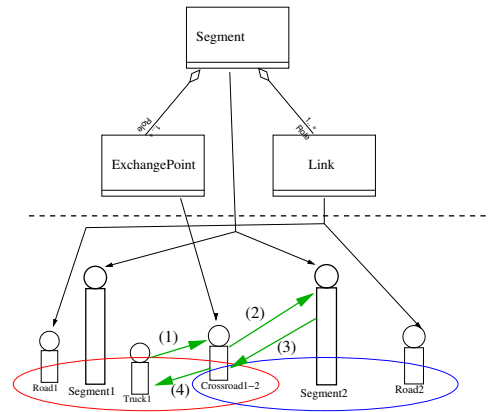

Fig. 3.

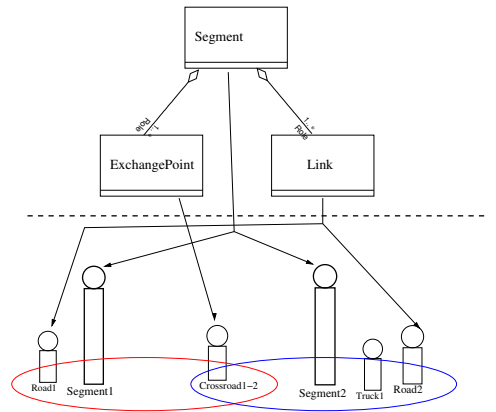

Fig. 4.

The dynamic choice of the environment granularity level during the simulation must be transparent for the agents. In order to do this, agents use our holonic framework and specifically ExchangePoint holons which enables the communication between holons of the same level and connected in the plant topology. Figure 3 describes the sequence of messages exchanged between the ExchangePoint, a vehicle and the Segment's Head. The truck agent is moving along segment 1 and requests the exchange point to forward a merging request. The exchange point forwards the request and receives a reply. The reply is then forwarded to the truck. If the reply is positive the truck can merge with the 
segment 2 holon as shown in figure 4. These interaction sequences are a mean to represent the influence/reaction model [6]. Indeed, the agent emits influences in asking to merge with a specific holon. The environment is able to determine the eventual answer according to jams or environment properties.

Each level stores pertinent information about the topology, characteristics and environment laws such as adjacent links, road signs, etc. These different granularity levels can coexist during a simulation. The advantages of this approach is threefold. First, it enables the decomposition of the complexity of the environment in an holarchy of components with only important aspects at each level. Second during the simulation the pertinent level of detail can be automatically chosen to be more efficient.

Finally, in order to support real-time application with high density of agents, the environmental rules can be

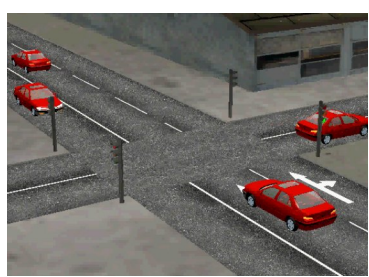

Fig. 5: View of different crossroads and buildings assigned by zone or region. This lets us regulate the behavior of the system according to the simulation requirements. For instance, in a Virtual Reality simulation, a high level of precision is required in the surroundings of the avatar ${ }^{1}$. On the other hand, in distant regions, certain environmental rules can be relaxed or annulled, such as collision detection.

\section{Related Works and Conclusion}

In this paper we have presented an approach for the modeling of environments for multi-agent based simulation. The modeling is based upon holonic concepts. The environment is represented as an holarchy. Each holon models an environmental component, which may be decomposed in sub-entities. This offers a modular and flexible representation for large scale environments.

We also discuss some of the advantages of this approach when compared to a global representation. Using this model, we have simulated the traffic within the PSA plant and we have observed emergent properties of the plant such as functional exchange between buildings, traffic congestion, jams, etc.

Hierarchical representation of the environment has already been successfully applied in several applications, mainly in the field of Virtual Reality $[4,5]$ and GIS [12]. The main difference between these approaches and ours is that we see the components as active entities.

Considering the importance of traffic flow simulation, it is not surprising that a vast number of models and simulators can be found. The Smarttest project [16] provides an extensive survey of microscopic traffic simulators. These simulators were conceived with different purposes (Urban or Free way traffic, Automated Highway Systems, etc), however, to our knowledge, the environment is not seen as a first-class entity of the model.

Future investigations will consider new simulation cases in order to extract a methodology from our approach. We are developing a formal specification model

\footnotetext{
${ }^{1}$ Virtual representation of the human user.
} 
of the concepts[14]. Such a specification may enable verification and validation. The development of an API in JAVA using the MadKit platform [7] is currently undertaken.

\section{References}

1. Emmanuel Adam, Rene Mandiau, and Christophe Kolski. Une Méthode de modelisation et de conception d'organizations Multi-Agents holoniques, chapter 2, pages 41-75. Hermes, 2002.

2. H. Van Brussel, J. Wyns, P. Valckenaers, L. Bongaerts, and P. Peeters. Reference architecture for holonic manufacturing systems: Prosa, 1998.

3. H.-J. Bürckert, K. Fischer, and G.Vierke. Transportation scheduling with holonic mas - the teletruck approach. In Proceedings of the Third International Conference on Practical Applications of Intelligent Agents and Multiagents, 1998.

4. Stéphane Donikian. How introduce life in virtual environments: a urban environment modeling system for driving simulation. Technical report, Institut de Recherche en Informatique de Toulouse, octobre 1996.

5. N. Farenc, R. Boulic, and D. Thalmann. An informed environment dedicated to the simulation of virtual humans in urban context. In Proc. Eurographics '99, Milano, Italy, 1999.

6. Jacques Ferber and Jean-Pierre Müller. Influences and reaction: a model of situated multiagent systems. In ICMAS'96, december 1996.

7. Olivier Gutknecht and Jacques Ferber. The MADKIT agent platform architecture. In Agents Workshop on Infrastructure for Multi-Agent Systems, pages 48-55, 2000.

8. Arthur Koestler. The Ghost in the Machine. Hutchinson, 1967.

9. Fabien Michel. Formalisme, méthodologie et outils pour la modélisation et la simulation de systèmes multi-agents. PhD thesis, Université de Montpellier II, 2004.

10. Fabien Michel, Abdelkader Gouaich, and Jacques Ferber. Weak interaction and strong interaction in agent based simulations. In The 4 th Workshop on Multi-Agent Based Simulation MABS'03 at AAMAS 2003, Melbourne, Australia, july 2003. to appear in a LNCS volume.

11. James Odell, H. Van Dyke Parunak, Mitch Fleischer, and Sven Breuckner. Modeling agents and their environment. In F. Giunchiglia, James Odell, and Gerhard Weiss, editors, Agent-Oriented Software Engineering (AOSE) III, volume 2585 of Lecture Notes on Computer Science, pages 16-31, 2002.

12. L.S.C. Pun-Cheng. A new face-entity concept for modeling urban morphology. Journal of Urban and Regional Information Systems Association, 12(3), 2000.

13. Sebastian Rodriguez, Vincent Hilaire, and Abderrafiâa Koukam. Towards a methodological framework for holonic multi-agent systems. In Fourth International Workshop of Engineering Societies in the Agents World, Imperial College London, UK (EU), 29-31 Octubre 2003.

14. Sebastian Rodriguez, Vincent Hilaire, and Abderrafiâa Koukam. Fomal specification of holonic multi-agent system framework. In Intelligent Agents in Computing Systems - The Agent Days in Atlanta, Lecture Notes in Computer Science. Springer-Verlag, 2005. to appear.

15. Herbert A. Simon. The Science of Artificial. MIT Press, Cambridge, Massachusetts, 3rd edition, 1996.

16. Project Smarttest. Smartest. final report. Technical report, Smartest Project, 2000. 